\title{
Measurement of Human Lower Limb Orientations and Ground Reaction Forces Using Wearable Sensor Systems
}

\author{
Tao Liu*, Yoshio Inoue, Kyoko Shibata and Rencheng Zheng \\ Department of Intelligent Mechanical Systems Engineering \\ Kochi University of Technology \\ 185 Miyanokuchi, Tosayamada-cho, Kochi Japan 782-8502 \\ liu.tao@kochi-tech.ac.jp
}

\begin{abstract}
This paper presents a study on quantitative dynamics analysis of human lower limb using developed wearable sensor systems that can measure reaction force and detect the following gait phases: initial contact, loading response, mid stance, terminal stance, pre-swing, initial swing, mid swing and terminal swing. Since conventional camera-based motion analysis system and reaction force plate system require costly devices, vast space as well as time-consuming calibration experiments, the wearable sensor-based system is much cheaper. Gyroscopes and two-axis accelerometers are incorporated in this wearable sensor system. The former are attached on the surface of the foot, shank and thigh to measure the angular velocity of each segment, and the latter are used to measure inclination of the attached leg segment (shank) in every single human motion cycle for recalibration. Ground reaction forces during human walking are synchronously measured using a wearable force sensor integrated in a shoes mechanism. Finally, experiment has been performed to compare the measurement results from the wearable sensor system with the data obtained from an optical motion analysis system and a force plate. The results showed that the measurement of human lower limb orientations and reaction forces for human dynamics analysis could be reliably implemented using the wearable sensor systems.
\end{abstract}

Index Terms - human dynamics analysis; wearable sensor system; gait phases; ground reaction force.

\section{INTRODUCTION}

The integration of 3-D motion measurement using multicamera systems and reaction force measurement using force plates has been successfully developed for tracking human body parts and performing dynamics analysis of their physical behaviors in a complex environment [1]. However, the optical motion analysis method needs considerable workspace and high-speed graphic signal processing devices, and using this analysis method, the devices are expensive, and precalibration experiments and offline analysis of recorded pictures are especially complex and time-consuming. Therefore, this method is limited to laboratory research, and can't be used in applications. Moreover, the human body is composed of many highly flexible segments, and the upperbody motion of humans is especially complicated in terms of accuracy calculations.

Thus, cheaper and more comfortable gait analysis devices with multi-sensor system, including force sensitive resistors, inclinometer, goniometers, gyroscopes and accelerometers, were proposed for human gait phases analysis and human motion analysis. Many researchers have used singleaccelerometer or multi-accelerometer systems for human gait phase detection or assessment, and these works possibly find applications in clinical and robotics research. Gait phases detection from accelerometer data was implemented to distinguish between stance and swing phase [2], but large disturbances in the acceleration signal may affect real-time precision in clinical applications. The quantitative analysis of human motion was also investigated in [3], and using assembled multi-axis accelerometer sensors, a measurement system was developed for estimation of 3-D position and orientation of a body segment, but large estimate errors from offset error in the accelerometer and inaccuracy in the orientation of the individual accelerometer's active axis make this system unsuitable for quantitative body segment motion analysis in biomechanical applications.

Accelerometer signals do not contain information about rotation around the vertical axis and therefore do not give a complete description of human motion. In [4], Tong and Grant proposed a measurement device using two gyroscopes, one placed on the thigh and the other on the shank, which can estimate knee rotation angle during walking. This system can detect different phases of human walking, but the quantitative analysis of leg motion was not completed in this study. Ion P.I. Pappas al. in [5] used a detection system consisting of three force-sensitive resistors, which measure the force loads on a shoe insole, and a gyroscope which measures the rotational velocity of the foot. The system detects the four gait phases accurately and reliably in real-time condition, but it was only designed for application to functional electrical stimulation.

A problem of the inertial sensors of gyroscopes and accelerometers is that they suffer from a fluctuating offset, which results from temperature change or small changes in the structure (mechanical wear). When accelerometers are used in clinical applications, a complex calibration procedure is impractical and misuse may be caused. Luinge proposed a Kalman filter that fuses tri-axial accelerometer and a tri-axial gyroscope signals for ambulatory recording of human body segment orientation [6].

In our past research [7] [8], a motion analysis system have been developed by combination of three gyroscopes that measure the rotational velocity of the foot, shank and thigh, and a two-axis accelerometer-chip that can detect twodirectional accelerations around the ankle during walking. In 
this paper, a wearable sensor system including motion sensor and reaction force sensor for quantitative lower limb dynamics analysis of human walking is introduced. An estimation algorithm based on kinematic restriction of human walking was developed to continuously correct errors of orientations obtained by mathematical integration of the angular velocity measured using gyroscopes. Analytical results including leg segment orientations and ground reaction forces obtained from the wearable sensor system were compared with the data obtained from a laboratory optical motion analysis system and a force plate.

\section{METHODS AND MATERIALS}

\section{A. Wearable Force Sensor and Motion Sensor Unit}

As shown in Fig.1 (a), an electrical baseboard was designed for integrating gyroscope (Murata ENC-03J, with dimensions of $15.5 \times 8.0 \times 4.3 \mathrm{~mm}$ and weight of $10 \mathrm{~g}$ ) and accelerometerchip (ADXL202). Gyroscope is used to measure angular velocities of leg segments of foot, shank and thigh $\left(\omega_{1}\right.$, $\omega_{2}$ and $\left.\omega_{3}\right)$. The sensitive axis is vertical to the medial-lateral plane so that the angular velocity in the sagittal plane can be detected. In local coordinates of three segments, sensitive axis of the gyroscopes is along y-axis, and the z-axis is along the leg-bone. A two-axis accelerometer is attached on the side of shank to measure two-direction accelerations along tangent direction of the x-axis $\left(a_{t}\right)$ and sagittal direction of the z-axis $\left(a_{r}\right)$. In this system, the data from the accelerometers are fused with data collected from the gyroscopes for cycle system calibration, based on the initial angular displacements of the attached leg segments. A new wearable force sensor has been developed based on the human dynamics analysis (Fig.1 (b)). The mass of the entire sensor system is about $0.5 \mathrm{~kg}$, and the whole dimensions are $115 \mathrm{~mm}$ in length, $115 \mathrm{~mm}$ in width and $35 \mathrm{~mm}$ in height. Moreover, a shoe mechanism integrating the motion sensor unit and the six-axis reaction force sensor was developed to measure ground reaction forces during human walking (Fig.1 (c)).

\section{B. Development of Wearable Sensor System}

As shown in Fig. 2, the wearable sensor system includes an eight-channel data recorder, a gyroscope-accelerometer combination unit, two gyroscope units and a wearable reaction force sensor. The two gyroscope units are attached on foot and thigh respectively, and the gyroscope-accelerometer combination unit is located on shank, near to ankle. The data recorder can be pocketed by the subject. The gyroscope measures the Coriolis acceleration, which is generated when a rotational angular velocity is applied to the oscillating piezoelectric bimorph. These inertial sensors can work under low energy consumption $(4.6 \mathrm{~mA}$ at $5 \mathrm{~V})$, and are accurate for ambulatory measurements. The signals from the gyroscopes and accelerometer are amplified and low-pass filtered (cutoff frequency: $25 \mathrm{~Hz}$ ) to remove electronic noise. The frequencies outside the pass-band are filtered out because they are invalid in study of human kinetics.

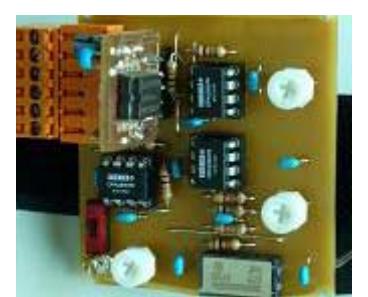

(a)

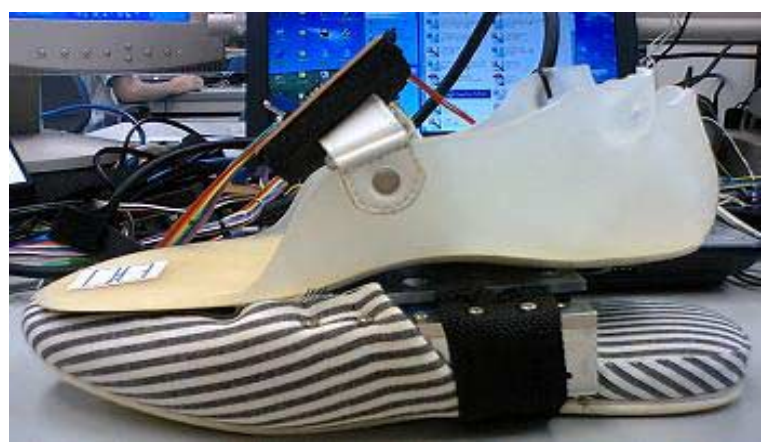

(c)

Fig. 1 Hardware devices of the sensor systems (a) Motion sensor unit with gyroscope and accelerometer; (b) Six-axis reaction force sensor; (c) A shoes mechanism integrating the motion sensor unit and the six-axis reaction force sensor.

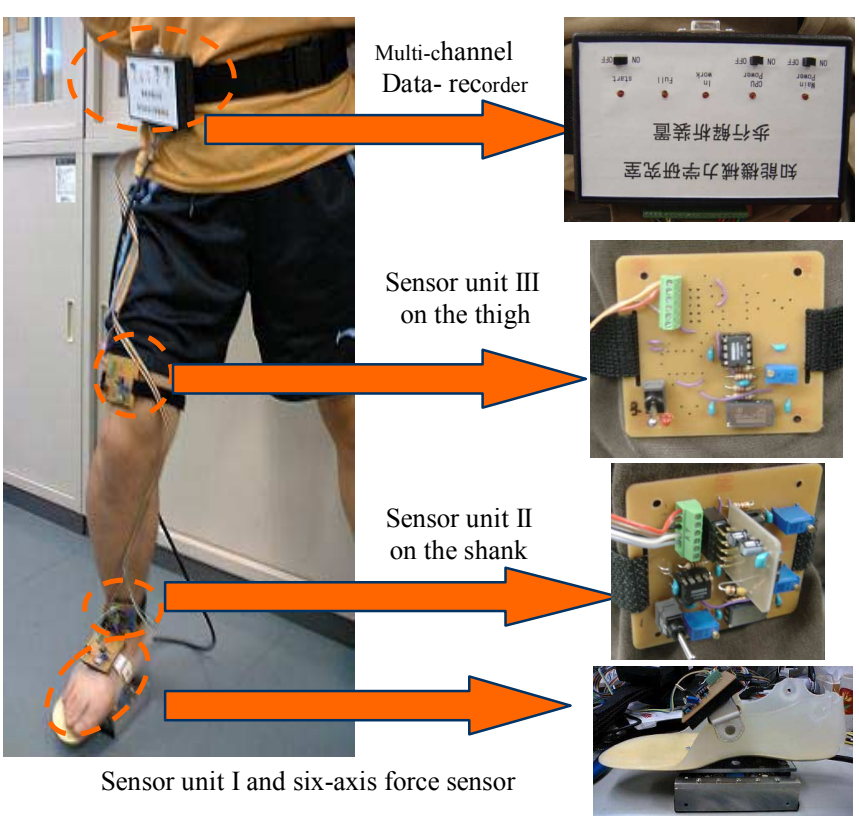

Fig. 2 Wearable sensor systems. A strap system was designed for the binding between the sensor units and human body. The sensor unit is attached to the strap. During walking, the strap is tied around the limb to secure the position of the sensor unit.

The multi-channel data-recorder is specially designed for the wearable sensor system. A micro-computer PIC 
(16F877A) is used to develop the pocketed data recorder, and sampling data from the inertial sensors can be saved in a SRAM which can keep recording lasting five minutes. An offline motion analysis can be performed based on the sampled data saved in the SRAM to a personal computer through a RS232 communication module. Since gyroscope (ENC-03J), accelerometer-chip (ADXL202) and PIC system are all devices of low energy consumption, the wearable sensor system is powered using a battery of $300 \mathrm{mAh}$ (NiMH 30R7H).

\section{Methods: Intelligent CALIBRATION to DeCREASE DRIFT ERROR}

\section{A. Introduction of Gait Phase}

In the past, it was conventional to use normal events as the critical actions of separated gait phases. While this practice proved appropriate for the amputee, it often failed to accommodate the gait deviations of patients impaired by paralysis or arthritis. For example, the onset of stance customarily has been called as heel strike [5] [6]; yet the heel of a paralytic patient may never contact the ground or do so much later in the gait cycle. Similarly initial floor contact may be by the whole foot (foot flat), rather than having forefoot contact occurs later, after a period of heel-only support. To avoid these difficulties and other areas of confusion, the Rancho Los Amigos gait analysis committee developed a generic terminology for the functional phases of gait [9].

Analysis of human walking pattern by phases more directly identifies the functional significance of the different motions generated at the individual joints and segments. In this paper, a normal walking gait cycle is divided into eight different gait phases, i.e., initial contact, loading response, mid stance, terminal stance, pre-swing, initial swing, mid swing and terminal swing (as shown in Fig. 3). The detailed definition of the gait phases is described in [10].

In the following discussion we assume that the subject is viewed from the right lateral side and anticlockwise rotations are considered to be positive. $\theta_{f}, \theta_{s}$ and $\theta_{t}$ represent the inclination angles of the foot, shank and thigh with respect to gravity direction respectively. $\omega_{f}, \omega_{s}$ and $\omega_{t}$ represent the angular velocities of the foot, shank and thigh in the lateral plane respectively. Finally, $\varepsilon_{\theta}, \varepsilon_{\omega}$ and $\varepsilon_{a}$ represent the small threshold values for the detection of close to zero angle displacements, angular velocities and accelerations, respectively.
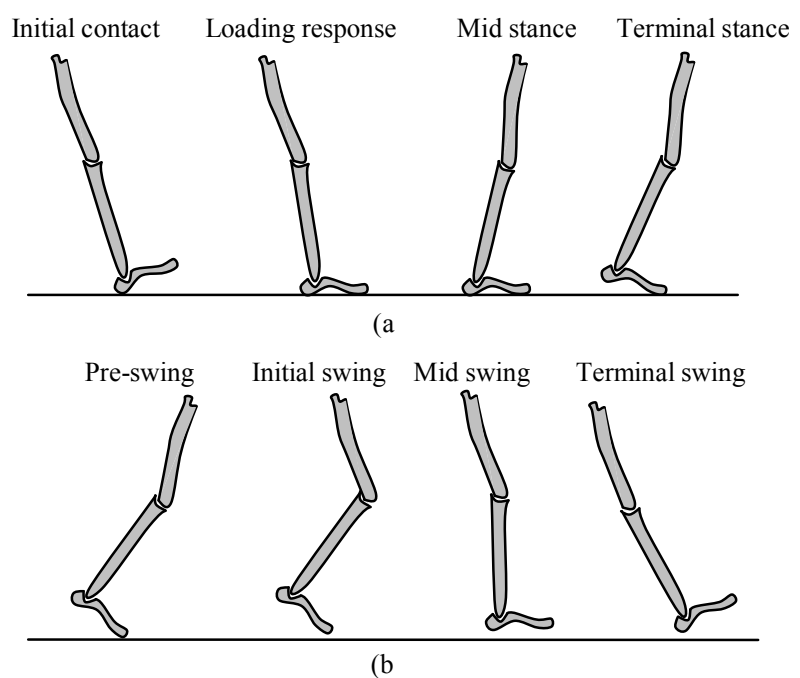

Fig. 3 Gait phase of normal walking. (a) Gait phases of stance period. (b) Gait phases of swing period.

\section{B. Gait Phase Detection Algorithm}

In the following discussion, we assume that the subject is viewed from the right lateral side and anticlockwise rotations are considered positive. $\theta_{f}, \theta_{s}$ and $\theta_{t}$ represent the inclination angles of the foot, shank and thigh with respect to gravity direction respectively. We also define $\theta_{f 0}, \theta_{s 0}$ and $\theta_{t 0}$ as initial (neutral) orientation angle. $\omega_{f}, \omega_{s}$ and $\omega_{t}$ represent the angular velocities of the foot, shank and thigh in the lateral plane respectively. Finally, $\varepsilon_{\theta}, \varepsilon_{\omega}$ and $\varepsilon_{a}$ represent the small threshold values for the detection of close to zero angle displacements, angular velocities and accelerations respectively.

A completed (normal) gait cycle is defined as follows:

1). $\mathrm{S}_{1}$ : Start of initial contact (end of terminal swing). The hip is flexed, the knee is extended $\left(\theta_{s}-\theta_{t}=\theta_{s 0}-\theta_{t 0}\right)$, and the ankle is dorsiflexed to neutral $\left(\theta_{f}-\theta_{s}=\theta_{f 0}-\theta_{s 0}\right)$. The inclinations of the leg segments are obtained by integrating the gyroscopes signal.

Sensor condition: $\omega_{f}=\varepsilon_{\omega}, \omega_{s}=\varepsilon_{\omega}$ and $\omega_{t}=\varepsilon_{\omega}$.

2). S2: Start of loading response (end of initial contact). Using the heel as a rocker, the knee is flexed for shock absorption $\left(\theta_{s}-\theta_{t}<\theta_{s 0}-\theta_{t 0}, \theta_{s}>0\right.$ and $\left.\theta_{t}>0\right)$. Sensor condition: $\omega_{f}<0, \omega_{s}<0$ and $\omega_{t}<0$.

3). S3: Start of mid stance (end of loading response). In this phase, the limb advances over the stationary foot by ankle dorsiflexion (ankle rocker) while the knee and hip extend $\left(\theta_{s}-\theta_{t}=\varepsilon_{\theta}\right)$.

Sensor condition: $\omega_{f}=\varepsilon_{\omega}, \omega_{s}<0$ and $\omega_{t}<0$.

4). S4: Start of terminal stance (end of mid stance). The terminal stance begins with heel rise and continues until 
the other foot strikes the ground, in which the heel rise and the limb advance over the forefoot rocker $\left(\theta_{s}-\theta_{t}=\theta_{s 0}-\theta_{t 0}, \theta_{f}<0, \theta_{s}<0\right.$ and $\left.\theta_{t}<0\right)$.

Sensor condition: $\omega_{f}<0, \omega_{s}<0$ and $\omega_{t}<0$.

5). S5: Start of pre-swing (end of terminal stance). The limb responds with increased ankle plantar flexion $\left(\theta_{f}<0\right)$, greater knee flexion $\left(\theta_{s}-\theta_{t}<0\right)$ and loss of hip extension.

Sensor condition: $\omega_{f}<0, \omega_{s}<0$ and $\omega_{t}<0$.

6). S6: Start of initial swing (end of pre-swing). In this phase, the foot is lifted and limb advanced by hip flexion and increased knee flexion $\left(\theta_{s}-\theta_{t}<0\right)$.

Sensor condition: $\omega_{f}>0, \omega_{s}>0$ and $\omega_{t}>0$.

7). S7: Start of mid swing (end of initial swing). The knee is allowed to extend in response to gravity while the ankle continues dorsiflexion to neural $\left(\theta_{f}-\theta_{s}<0, \theta_{t}>0\right.$ and $\left.\theta_{s}<\varepsilon_{\theta}\right)$.

Sensor condition: $\omega_{f}>0, \omega_{s}>0$ and $\omega_{t}>0$.

8). S8: Start of terminal swing (end of mid swing). Limb advancement is completed as the leg (shank) moves ahead of the thigh. In this phase the limb advancement is completed by knee extension, and the hip maintains its earlier flexion $\left(\theta_{f}-\theta_{s}=\theta_{f 0}-\theta_{s 0}\right)$, and the ankle remains dorsiflexed to neural.

Sensor condition: $\omega_{f}-\omega_{s}=\varepsilon_{\omega}$ and $\omega_{t}>0$.

The motion analysis system with intelligent calibration for leg segment quantitative leg-motion analysis is developed. According to the above quantitative assessment of the eight gait phases, if the leg segments' orientations ( $\theta_{f}, \theta_{s}$ and $\left.\theta_{t}\right)$ can not be accurately obtained and combined with the signals of the sensor units $\left(\omega_{f}, \omega_{s}\right.$ and $\left.\omega_{t}\right)$, it is almost impossible that all the gait phases are effectively detected

\section{Quantitative leg- motion Analysis}

The loop frequency of the phase record is $100 \mathrm{~Hz}$, equal to the sensors sampling frequency, and the number of sampling time point is counted in an integer value $\mathrm{i}(\mathrm{i}=1,2,3 \ldots)$. The orientation of leg segment $(\theta(i))$ can be calculated by integration of the angular velocity $(\omega(i))$ of leg segment $((1)$ and (2)), which is directly measured using the wearable sensor unit. The inclination of shank and thigh is set to be zero in the initial period, while the inclination of foot is set to be $90^{\circ}$ at start. However, the gyroscope in the sensor unit is a kind of inertial sensor that is affected by drift errors when it is worn on human body, thus the integral calculation in (1) may produce accumulated errors in a multi-step walking motion analysis.

$$
\theta(i)=\theta(i-1)+(\omega(i-1)+\omega(i)) \Delta t / 2
$$

where

$$
\theta(0)=\theta_{0} ; \quad \mathrm{i}=1,2,3 \ldots
$$

We define the gait cycle (walking gait cycle number $\mathrm{k}=1$, $2 . .$. ) as the period from one stance phase of the foot to the next stance phase of one foot. In every walking cycle, the time points of transition from loading response phase to mid stance phase, and transition from pre-swing to initial swing phase are defined as $T_{41}(k), T_{42}(k), T_{43}(k)$ and $T_{44}(k)$ respectively. Based on a pre-analysis of gait phase, the human motion analysis is implemented by calculating body segments' angular displacements using inertial sensors of gyroscopes and accelerometers. As shown in Fig. 4 and Fig. 5, the mid stance phase can be primarily detected just using gyroscope signals and raw integration results of gyroscope signals from the three sensor units $\left(\omega_{t}<0, \omega_{f}=\varepsilon_{\omega}, \omega_{s}<0\right.$ and $\left.\theta_{s}<0\right)$.

Moreover, it can be seen that the rotational angular velocities of the shank and thigh are quite small in later interval of this phase, because the ankle is in state of dorsiflexion, and the shank rotational velocity is limited. Therefore, the accelerometer can be used for inclination measurement with respect to gravity acceleration, when shank's sagittal direction $A_{r}$ (3) and $A_{t}$ (4) are mainly affected by the gravity acceleration's projection. Hence, the cycle calibration can be performed by measuring initial angular orientation of the attached segment (shank) using (5), and foot orientation $\left(\theta_{f}^{m}=0\right)$ and thigh orientation $\left(\theta_{t}^{m}=\theta_{s}{ }^{m}\right)$ are the initial calibration quantities for calculation of foot and thigh orientation. Integral calculations are performed in every gait cycle, which can reduce the accumulated errors in the longtime walking experiments.

$$
\begin{gathered}
A_{r}=-g \cdot \cos \left(\theta_{s}{ }^{m}\right)+D \cdot\left({\omega_{s}}^{m}\right)^{2} \\
A_{t}=-g \cdot \sin g\left(\theta_{s}{ }^{m}\right)+D \cdot \dot{\omega}_{s}{ }^{m} \\
\theta_{s}{ }^{m}=\arctan \left(A_{t} / A_{r}\right)
\end{gathered}
$$

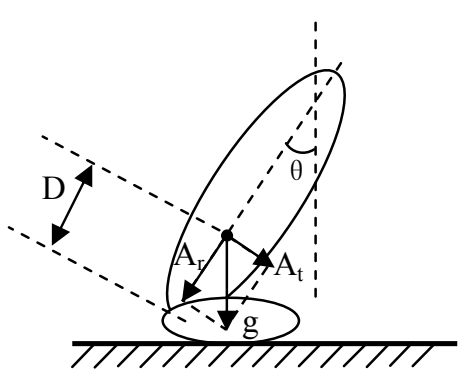

Fig. 4 Shank angle in Mid stance phase 


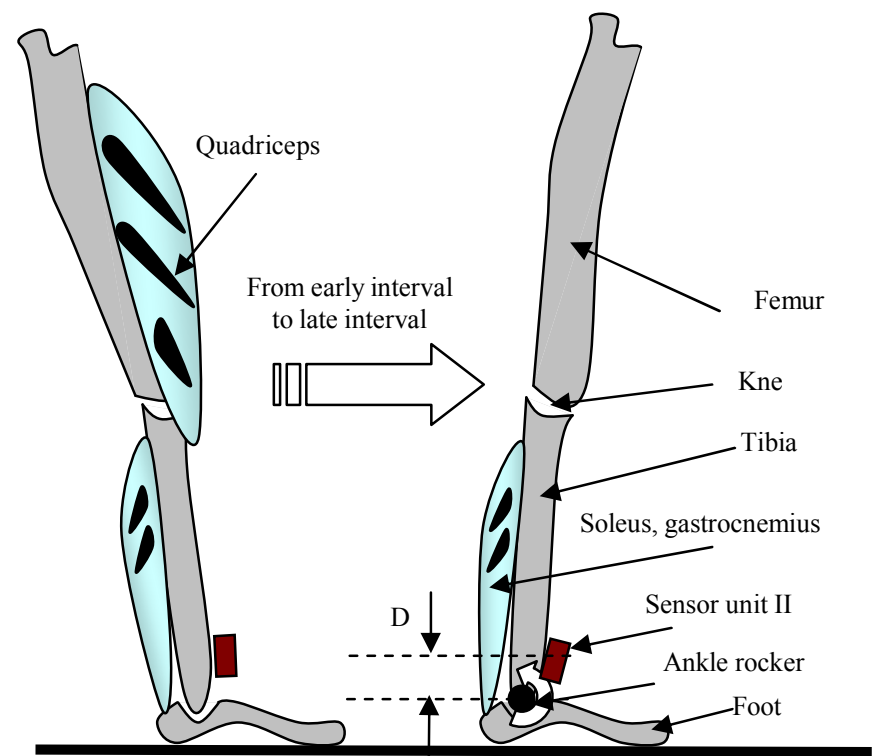

Fig. 5 Mid stance phase including early interval and late interval. The orientation calibration is implemented in late interval of mid stand. Early interval has body over mid foot with climb vertical, ankle neutral and foot flat, in which quadriceps and soleus muscles are in activity. Later interval has body over forefoot with continued heel contact, while ankle is in state of dorsiflexion which limit shank rotational velocity. A small distance between sensor unit II and ankle rocker is denoted using D (it is about $50 \mathrm{~mm}$ ). Moreover in later interval, soleus and gastrocnemius are only extensor muscles around tibia, which produces least vibration effect on the accelerometer.

\section{EXPERIMENT: LOW LIMB DYNAMICS ANALYSIS}

The experiment of the sensory system for leg motion analysis is implemented in the following three steps. Firstly, the sensor devices are worn on the subject's leg to measure 2D motion of the foot, shank and thigh, and the sensors' data are saved in the pocketed data recorder. Secondly when the human motion record is finished, the data in the data recorder will be fed into personal computer through serial port (RS232), then leg-motion data is prepared for the offline motion analysis computing. Finally, the leg motion analysis is performed to estimate the leg segments' angular orientations.

\section{A. Quantitative Gait Phase Analysis}

When the experiments data are recorded in the personal computer, and the quantitative human motion analysis was completed to calculate leg segments orientation angles and angular velocities, an off-line analysis was made to analyze the gait during walking. The inertial sensor of gyroscopes and accelerometers' output signals are sensitive to the external noises (for example temperature effect), and the drift of these inertial sensors reduces the measurement precision during long time testing. In this study, an intelligent calibration method is used to calculated segment orientation angle to improve precision of the estimation of orientation. An inference system is introduced to detect the eight-gait phase using gyroscope signals and orientation angles estimated in human motion analysis, which is robust to the noise from the inertial sensor of gyroscopes. The inference system was designed using MATLAB software. The signals form the three gyroscopes and the three leg segments' orientation angles were defined as six input vectors of this inference system. The inference rules were designed from the algorithm of gait phase detection introduced in Section II. As shown in Fig. 6, a human walking gait cycle is separated into eight phases according to this inference system.

\section{B. Experimental Verification}

To validate the sensor system performance comparison of the quantitative results of the sensor system and the measurements obtained with a commercial optical motion analysis system Hi-DCam (NAC image technology. Japan) was performed. The motion analysis system (Hi-DCam) tracked and measured the 3-D trajectories of retro-reflective markers placed on the subject's body, as shown in Figs. 7. The cameras with sampling frequency of $100 \mathrm{~Hz}$ were used to track the marker positions with accuracy of $1 \mathrm{~mm}$. A force plate of EFP-S-2KNSA12 was used as the reference sensor to validate the developed force sensor. In our experiment, these two sensor systems worked in the synchronized mode.

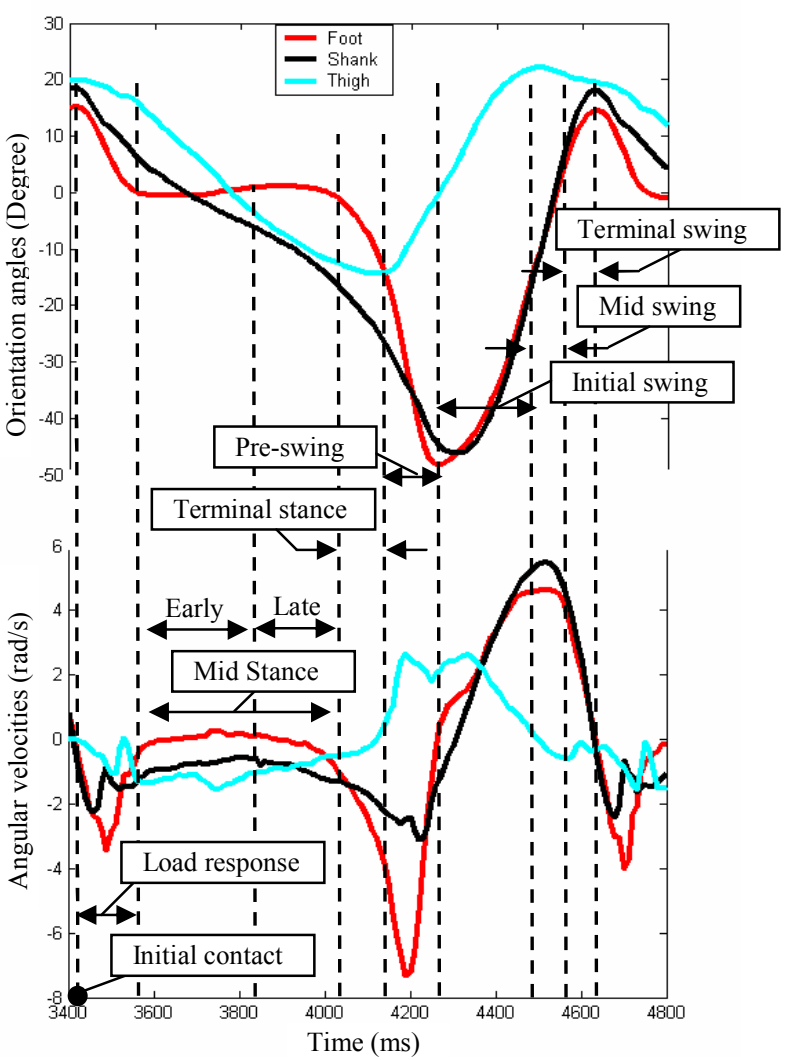

Fig. 6 Gait phase analysis using results of the quantitative limb motion analysis. The three gyroscopes' signals (angular velocities) and the three leg segments' orientation angles were used to analyze a healthy gait cycle which is divided into eight intervals of gait phases: initial contact, loading response, mid stance, terminal stance, pre swing, initial swing, mid swing and terminal swing. 

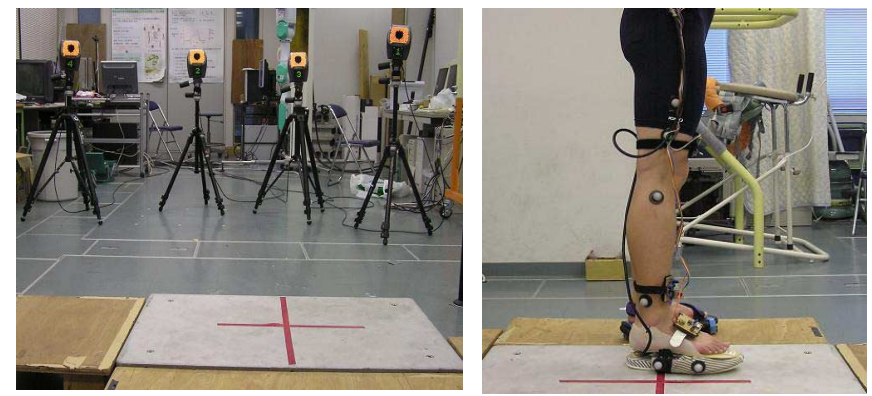

(a)

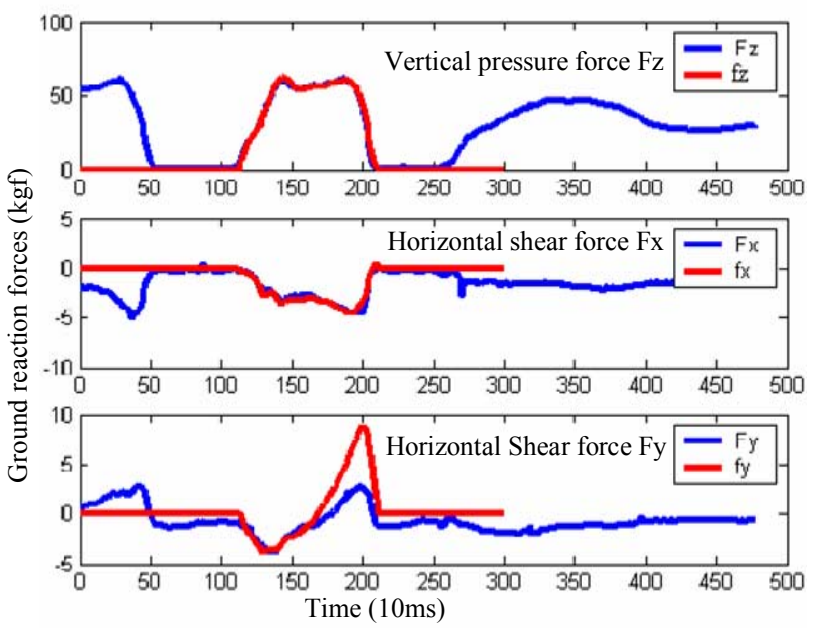

(b)

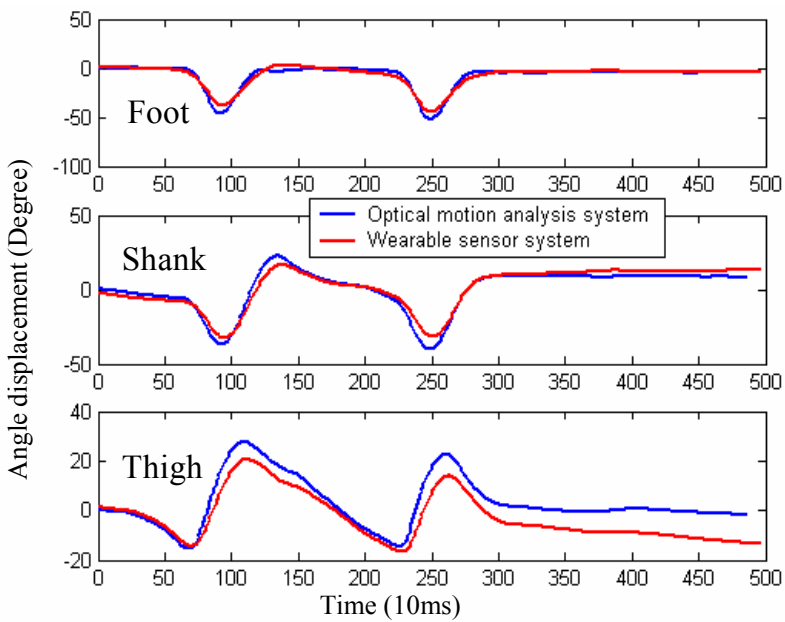

(c)

Fig. 7 Validation experimental study. (a)Experimental devices pictures and positions of the retro-reflective markers (b)Results of the validation experiments. Graphs show the comparisons between developed wearable sensor $(F)$ and the force plate (f) in the measurements of vertical force and horizontal forces. (c) Angle estimation results from the two motion analysis systems

\section{DISCUSSIONS AND CONCLUSIONS}

Measurement of human lower limb orientations and reaction forces for quantitative human dynamics analysis was implemented using the developed wearable sensor system. The results from the sensor system showed a high correlation to the results from the optical motion analysis system and the force plate in the multi-step walking validation experiment.

The muscular tensions are complex during walking, and the electromyogram (EMG) is a common method for this study. In next step of this study, we want to develop a wearable sensor system for estimation of muscular tensions instead of EMG. Analysis of human walking pattern by the eight-gait phases more directly identifies the functional significance (muscular tensions) of the different motions generated at the individual joints and segments. For example, in mid stance phase including early interval and late interval, the early interval has body over mid foot, in which quadriceps and soleus muscles are in activity; later interval has body over forefoot, in which soleus and gastrocnemius are only extensor muscles around tibia. We can use the developed wearable sensor system to estimate muscular tensions. The gait phase division will improve the precision of this method by providing constrain condition about the functional muscles for an optimum analysis [11].

Moreover, in the study of robotics, the wearable sensor systems will be applied for real-time control of a humanoid robot which may walk in the same phase as human walking.

\section{REFERENCES}

[1] Karaulova I.A., Hall P.M., Marshall A.D., "Tracking people in three dimensions using a hierarchical model," Image and Vision Computing, 2002, 20, pp.691-700.

[2] Bonato, P. "Wearable sensors/systems and their impact on biomedical engineering," IEEE Eng. Med. Biol. Mag., 2003, 22 (3) pp. 18-20.

[3] Giansanti D, Macellari V, Maccioni G, Cappozzo A. "Is it feasible to reconstruct body segment $3-\mathrm{D}$ position and orientation using accelerometric data?,” IEEE Trans. Biomed. Eng., 2003, 50 pp. 476-483.

[4] Tong K. and Granat H. M., "A practical gait analysis system using gyroscopes,” Med. Eng. Phys., 1999, 21, pp. 87-94.

[5] Ion P. I. Pappas, Milos R. Popovic, Thierry Keller, Volker Dietz, and Manfred Morari, "A reliable gait phase detection system," IEEE Trans. Rehab. Eng., 2001, 9 (2), pp. 113-125.

[6] Henk J. Luinge, Peter H. Veltink, "Inclination measurement of human movement using a 3-D accelerometer with autocalibration," IEEE Trans. Rehab. Eng., 2004, 12 pp. 112-121.

[7] Tao Liu, Yoshio Inoue, Kyoko Shibata, Xueyan Tang. A Wearable Inertial Sensor System for Human Motion Analysis. Proceedings of IEEE CIRA2005, Finland, pp. 409-413.

[8] Tao Liu, Yoshio Inoue, Kyoko Shibata, Haruhiko Morioka. "Development of Wearable Sensor Combinations for Human Lower Extremity Motion Analysis" Proceedings of the 2006 IEEE International Conference on Robotics and Automation, pp. 1655-1660.

[9] Pathokinesiology Department, Physical Therapy Department: "Observational Gait Analysis Handbook." CA, The professional staff association of rancho Los Amigos Medical Center, 1989.

[10] Jacquelin Parry. "Gait Analysis Normal and Pathological Function," Slack Incorporated, 1992.

[11] Ehara Y., Beppu M., Nomura S., and Kunimi Y., "Estimation of energy consumption during level walking," Biomechanisms, (in Japanese), 1990, 10, pp. 163-172. 